\title{
TRIANGULAR FEJÉR SUMMABILITY OF TWO-DIMENSIONAL WALSH-FOURIER SERIES
}

\author{
GYÖRGY GÁT AND USHANGI GOGINAVA
}

\begin{abstract}
It is proved that the operators $\sigma_{n}^{\triangle}$ of the triangular-Fejérmeans of a two-dimensional Walsh-Fourier series are uniformly bounded from the dyadic Hardy space $H_{p}$ to $L_{p}$ for all $4 / 5<p \leq \infty$.
\end{abstract}

\section{INTRODUCTION}

Lebesgue's 13 theorem is well known for trigonometric Fourier series: the Fejér means $\sigma_{n} f$ of $f$ converge to $f$ almost everywhere if $f \in L_{1}(\mathbb{T}), \mathbb{T}:=$ $[-\pi, \pi)$ (see also Zygmund [27]).

An analogous result for Walsh-Fourier series is due to Fine [1]. Later, Schipp [16] showed that the maximal operator $\sigma^{*}$ of the Fejér means of the one-dimensional Walsh-Fourier series is of weak type $(1,1)$, from which the a.e. convergence follows by standard arguments. Schipp's result implies by interpolation also the boundedness of $\sigma^{*}: L_{p}(G) \rightarrow L_{p}(G)$, where $1<p \leq$ $\infty$. This fails to hold for $p=1$, but Fujii [3] proved that $\sigma^{*}$ is bounded from the dyadic Hardy space $H_{1}(G)$ to the space $L_{1}(G)$ (see also Simon [18]). Fujii's theorem was extended by Weisz [20]. Namely, he proved that $\sigma^{*}$ is bounded from the martingale Hardy space $H_{p}(G)$ to the space $L_{p}(G)$ for $p>1 / 2$. Simon [19] gave a counterexample, which shows that this boundedness does not hold for $0<p<1 / 2$. In the endpoint case $p=1 / 2$, Weisz [22] proved that $\sigma^{*}$ is bounded from the Hardy space $H_{1 / 2}(G)$ to the space weak- $L_{1 / 2}(G)$. Goginava proved in [8] that the maximal operator of the Fejér means of the one dimensional Walsh-Fourier series is not bounded from the Hardy space $H_{1 / 2}(G)$ to the space $L_{1 / 2}(G)$.

Marcinkievicz [14] verified for two-dimensional trigonometric Fourier series that the Marcinkiewicz-Fejér means

$$
\sigma_{n}^{\square} f=\frac{1}{n} \sum_{j=0}^{n-1} S_{j}^{\square}(f)
$$

\footnotetext{
${ }^{0} 2010$ Mathematics Subject Classification $42 \mathrm{C} 10$.

Key words and phrases: two-dimensional Walsh system, triangular means, Hardy spaces, norm convergence.

Research was supported by project TÁMOP-4.2.2.A-11/1/KONV-2012-0051 and by Shota Rustaveli National Science Foundation grant no.13/06 (Geometry of function spaces, interpolation and embedding theorems)
} 
of a function $f \in L \log L(\mathbb{T} \times \mathbb{T})$ converge a.e. to $f$ as $n \rightarrow \infty$, where $S_{j}^{\square}(f)$ denotes the qubical partial sums of the Fourier series of $f$. Later Zhizhiashvili [25, 26] extended this result to all $f \in L_{1}(\mathbb{T} \times \mathbb{T})$.

An analogous result for two-dimensional Walsh-Fourier series is due to Weisz [21]. Moreover, he proved that the maximal operator $\sigma_{*}^{\square} f=\sup _{n \geq 1}\left|\sigma_{n}^{\square} f\right|$ is bounded from the dyadic martingale Hardy space $H_{p}(G \times G)$ to the space $L_{p}(G \times G)$ for $p>2 / 3$. The second author [11, 8] proved that the maximal operator $\sigma_{*}^{\square}$ is bounded from $H_{2 / 3}(G \times G)$ to weak $-L_{2 / 3}(G \times G)$ and is not bounded from $H_{2 / 3}(G \times G)$ to $L_{2 / 3}(G \times G)$.

Weisz [23, 24] studied the triangular partial sums and the Fejér means

$$
\sigma_{n}^{\triangle} f=\frac{1}{n} \sum_{j=0}^{n-1} S_{j}^{\triangle} f
$$

of the two-dimensional trigonometric Fourier series. This summability method is rarely investigated in the literature (see the references in [23]). In [12 it is proved that the maximal operator $\sigma_{\#}^{\triangle}:=\sup _{n}\left|\sigma_{2^{n}}^{\triangle} f\right|$ of the Fejér means of the triangular partial sums of the double Walsh-Fourier series is bounded from the dyadic Hardy space $H_{p}(G \times G)$ to the $L_{p}(G \times G)$ if $p>1 / 2$, is bounded from $H_{1 / 2}(G \times G)$ to the space weak- $L_{1 / 2}(G \times G)$ and it is not bounded from $H_{1 / 2}(G \times G)$ to $L_{1 / 2}(G \times G)$.

For triangular partial sums it is well-known [17] the operatos $S_{2^{A}}^{\triangle}$ are not unoformly bounded on $L_{p}$ for $1 \leq p \neq 2$.

It is proved that the operators $\sigma_{n}^{\triangle}$ of the triangular-Fejér-means of a twodimensional Walsh-Fourier series are uniformly bounded from the dyadic Hardy space $H_{p}$ to $L_{p}$ for all $4 / 5<p \leq \infty$.

The results for summability of quadratical partial sums of two-dimensional Walsh-Fourier series can be found in [7, 10, 4, 5].

\section{Definitions AND THE NOTATION}

Let $\mathbb{P}$ denote the set of positive integers, $\mathbb{N}:=\mathbb{P} \cup\{0\}$. Denote by $Z_{2}$ the discrete cyclic group of order 2 , that is $Z_{2}=\{0,1\}$, where the group operation is the modulo 2 addition and every subset is open. A Haar measure on $Z_{2}$ is given such that the measure of a singleton is $1 / 2$. Let $G$ be the complete direct product of the countable infinite copies of the compact groups $Z_{2}$. The elements of $G$ are of the form $x=\left(x_{0}, x_{1}, \ldots, x_{k}, \ldots\right)$ with $x_{k} \in\{0,1\}(k \in \mathbb{N})$. The group operation on $G$ is the coordinate-wise addition, the measure (denoted by $\mu$ ) and the topology are the product measure and topology. The compact Abelian group $G$ is called the Walsh group. A base for the neighborhoods of $G$ can be given by

$$
I_{n}(x):=I_{n}\left(x_{0}, \ldots, x_{n-1}\right):=\left\{y \in G: y=\left(x_{0}, \ldots, x_{n-1}, y_{n}, y_{n+1}, \ldots\right)\right\},
$$


where $I_{0}(x):=G$ and $x \in G, n \in \mathbb{N}$. These sets are called the dyadic intervals. Let $0=(0: i \in \mathbb{N}) \in G$ denote the null element of $G, I_{n}:=I_{n}(0)$ $(n \in \mathbb{N}), \bar{I}_{n}:=G \backslash I_{n}$. Denote

$$
2^{N} x:=\left(x_{N}, x_{N+1}, \ldots\right), x \in G .
$$

For $k \in \mathbb{N}$ and $x \in G$ denote

$$
r_{k}(x):=(-1)^{x_{k}} \quad(x \in G, \quad k \in \mathbb{N})
$$

the $k$-th Rademacher function. If $n \in \mathbb{N}$, then $n=\sum_{i=0}^{\infty} n_{i} 2^{i}$, where $n_{i} \in\{0,1\}$ $(i \in \mathbb{N})$, i.e. $n$ is expressed in the number system of base 2. Denote $|n|:=$ $\max \left\{j \in \mathbf{N}: n_{j} \neq 0\right\}$, that is, $2^{|n|} \leq n<2^{|n|+1}$.

The Walsh-Paley system is defined as the sequence of Walsh-Paley functions:

$$
w_{n}(x):=\prod_{k=0}^{\infty}\left(r_{k}(x)\right)^{n_{k}}=r_{|n|}(x)(-1) \sum_{k=0}^{|n|-1} n_{k} x_{k} \quad(x \in G, \quad n \in \mathbb{P}) .
$$

The Walsh-Dirichlet kernel is defined by

$$
D_{n}(x)=\sum_{k=0}^{n-1} w_{k}(x), D_{0}(x)=0 .
$$

Recall that $([17])$

$$
\begin{aligned}
& D_{2^{n}}(x)= \begin{cases}2^{n} & \text { if } x \in I_{n}, \\
0 & \text { if } x \in \bar{I}_{n}\end{cases} \\
& D_{n}(x)=-w_{n^{(i+1)}}(x)\left(\sum_{r=0}^{i-1} n_{r} 2^{r}-n_{i} 2^{i}\right) \text { for } x \in I_{i} \backslash I_{i+1} .
\end{aligned}
$$

In this paper we consider the double system $\left\{w_{i}(x) w_{j}(y): i, j \in \mathbb{N}\right\}$ on $G \times G$.

The rectangular partial sums of the 2-dimensional Walsh-Fourier series are defined as

$$
S_{M, N} f(x, y):=\sum_{i=0}^{M-1} \sum_{j=0}^{N-1} \widehat{f}(i, j) w_{i}(x) w_{j}(y),
$$

where the number

$$
\widehat{f}(i, j)=\int_{G \times G} f(x, y) w_{i}(x) w_{j}(y) d \mu(x, y)
$$

is said to be the $(i, j)$ th Walsh-Fourier coefficient of the function $f$. Denote

$$
S_{M}^{\square} f(x, y):=S_{M, M} f(x, y) .
$$


The triangular partial sums of the 2-dimensional Walsh-Fourier series are defined as

$$
S_{k}^{\triangle} f(x, y):=\sum_{i=0}^{k-1} \sum_{j=0}^{k-i-1} \widehat{f}(i, j) w_{i}(x) w_{j}(y) .
$$

Denote

$$
D_{k}^{\square}(x, y):=D_{k}(x) D_{k}(y)
$$

and

$$
D_{k}^{\triangle}(x, y):=\sum_{i=0}^{k-1} \sum_{j=0}^{k-i-1} w_{i}(x) w_{j}(y) .
$$

The norm (or the quasinorm) of the space $L_{p}(G \times G)$ is defined by

$$
\|f\|_{p}:=\left(\int_{G \times G}|f(x, y)|^{p} d \mu(x, y)\right)^{1 / p} \quad(0<p \leq \infty) .
$$

The space weak- $L_{p}(G \times G)$ consists of all measurable functions $f$ for which

$$
\|f\|_{\text {weak }-L_{p}(G \times G)}:=\sup _{\lambda>0} \lambda \mu(|f|>\lambda)^{1 / p}<+\infty .
$$

The $\sigma$-algebra generated by the dyadic 2-dimensional $I_{k}(x) \times I_{k}(y)$ cubes of measure $2^{-k} \times 2^{-k}$ will be denoted by $F_{k}(k \in \mathbb{N})$. Denote by $f=$ $\left(f^{(n)}, n \in N\right)$ one-parameter martingales with respect to $\left(F_{n}, n \in N\right)$ (for details see e.g. [22]). The maximal function of a martingale $f$ is defined by

$$
f^{*}=\sup _{n \in N}\left|f^{(n)}\right| \text {. }
$$

In case $f \in L_{1}(G \times G)$, the maximal function can also be given by

$$
\begin{gathered}
f^{*}(x, y)=\sup _{n \geq 1} \frac{1}{\mu\left(I_{n}(x) \times I_{n}(y)\right)}\left|\int_{I_{n}(x) \times I_{n}(y)} f(s, t) d \mu(s, t)\right|, \\
(x, y) \in G \times G .
\end{gathered}
$$

For $0<p<\infty$ the martingale Hardy space $H_{p}(G \times G)$ consists of all martingales for which

$$
\|f\|_{H_{p}}:=\left\|f^{*}\right\|_{p}<\infty .
$$

If $f \in L_{1}(G \times G)$, then it is easy to show that the sequence $\left(S_{2^{n}, 2^{n}}(f): n \in \mathbf{N}\right)$ is a martingale. If $f$ is a martingale, that is $f=\left(f^{(0)}, f^{(1)}, \ldots\right)$, then the Walsh-Fourier coefficients must be defined in a little bit different way:

$$
\widehat{f}(i, j)=\lim _{k \rightarrow \infty} \int_{G \times G} f^{(k)}(x, y) w_{i}(x) w_{j}(y) d \mu(x, y) .
$$

The Walsh-Fourier coefficients of $f \in L_{1}(G \times G)$ are the same as the ones of the martingale $\left(S_{2^{n}, 2^{n}}(f): n \in \mathbb{N}\right)$ obtained from $f$. 
For $n \in \mathbb{P}$ and a martingale $f$ the Marcinkiewicz-Fejér means and triangular Fejér means of order $n$ of the 2-dimensional Walsh-Fourier series of a function $f$ is given by

$$
\sigma_{n}^{\square} f(x, y)=\frac{1}{n} \sum_{j=0}^{n-1} S_{j}^{\square} f(x, y)
$$

and

$$
\sigma_{n}^{\triangle} f(x, y)=\frac{1}{n} \sum_{j=0}^{n-1} S_{j}^{\triangle} f(x, y),
$$

respectively. It is easy to show that

$$
\sigma_{n}^{\square} f(x, y)=\int_{G \times G} f(s, t) K_{n}^{\square}(x+s, y+t) d \mu(s, t)
$$

and

$$
\sigma_{n}^{\triangle} f(x, y)=\int_{G \times G} f(s, t) K_{n}^{\triangle}(x+s, y+t) d \mu(s, t),
$$

where

$$
K_{n}^{\square}(x, y):=\frac{1}{n} \sum_{j=0}^{n-1} D_{j}^{\square}(x, y)
$$

and

$$
K_{n}^{\triangle}(x, y):=\frac{1}{n} \sum_{j=0}^{n-1} D_{j}^{\triangle}(x, y) .
$$

We can write

$$
\begin{aligned}
K_{n}^{\triangle}(x, y) & =\frac{1}{n} \sum_{k=0}^{n-1} D_{k}^{\triangle}(x, y) \\
& =\frac{1}{n} \sum_{k=1}^{n-1} \sum_{i=0}^{k-1} \sum_{j=0}^{k-i-1} w_{i}(x) w_{j}(y) \\
& =\frac{1}{n} \sum_{k=1}^{n-1} \sum_{i=0}^{k-1} w_{i}(x) D_{k-i}(y) \\
& =\frac{1}{n} \sum_{k=1}^{n-1} \sum_{i=1}^{k} w_{k-i}(x) D_{i}(y) \\
& =\frac{1}{n} \sum_{i=1}^{n-1} \sum_{k=i}^{n-1} w_{k-i}(x) D_{i}(y) \\
& =\frac{1}{n} \sum_{i=1}^{n-1} D_{n-i}(x) D_{i}(y) .
\end{aligned}
$$


A bounded measurable function $a$ is a $p$-atom if there exists a dyadic 2dimensional cube $I$ such that

a) $\int_{I} a d \mu=0$

b) $\|a\|_{\infty} \leq \mu(I)^{-1 / p}$;

c) $\operatorname{supp} a \subset I$.

An operator $T$ which maps the set of martingale into the collection of measurable functions will be called $p$-quasi-local if there exists a constant $c_{p}>0$ such that for evey $p$-atom $a$

$$
\int_{G \times G \backslash I}|T a|^{p} \leq c_{p}<\infty,
$$

where $I$ is the support of the atom.

\section{Formulation of MAin RESUlts}

Theorem 1. If $4 / 5<p \leq \infty$, then the operators $\sigma_{n}^{\triangle}$ are uniformly bounded from the Hardy space $H_{p}(G \times G)$ to the space $L_{p}(G \times G)$.In particular, if $f \in H_{p}(G \times G)$, then

$$
\sup _{n}\left\|\sigma_{n}^{\triangle} f\right\|_{p} \leq c_{p}\|f\|_{H_{p}}
$$

\section{Auxiliary proposition}

We shall need the following lemma (see [22]).

Lemma 1. Suppose that the operator $T$ is $\sigma$-sublinear and $p$-quasi-local for each $0<p_{0}<p \leq 1$. If $T$ is bounded from $L_{\infty}(G \times G)$ to $L_{\infty}(G \times G)$, then

$$
\|T f\|_{p} \leq c(p)\|f\|_{H_{p}} \quad\left(f \in H_{p}(G \times G)\right)
$$

for every $0<p_{0}<p<\infty$.

Lemma 2. Let $p \in(1 / 2,1]$. Then

$$
\int_{G}\left(\sup _{1 \leq n \leq 2^{N}}\left|\sum_{j=1}^{n} D_{j}(x)\right|\right)^{p} d \mu(x) \leq c_{p} 2^{N(2 p-1)} .
$$

The proof can be found in [9].

Lemma 3. Let $p \in(1 / 2,1]$. Then

$$
\int_{G} \sup _{1 \leq n \leq 2^{N}}\left|\sum_{k=1}^{n} D_{k}(x)(n-k+1)\right|^{p} d \mu(x) \leq c_{p} 2^{N(3 p-1)} .
$$

Proof of Lemma 3. Applying Lemma 2 and Abel's transformation we obtain

$$
\int_{G} \sup _{1 \leq n \leq 2^{N}}\left|\sum_{k=1}^{n} D_{k}(x)(n-k+1)\right|^{p} d \mu(x)
$$




$$
\begin{gathered}
\text { TRIANGULAR FEJÉR SUMMABILITY } \\
\leq c_{p} 2^{N p} \int_{G} \sup _{1 \leq n \leq 2^{N}}\left|\sum_{k=1}^{n} D_{k}(x)\right|^{p} d \mu(x) \\
+c_{p} \int_{G}\left(\sup _{1 \leq n \leq 2^{N}} \sum_{k=1}^{n}\left|\sum_{l=1}^{k} D_{k}(x)\right|\right)^{p} d \mu(x) \\
\leq c_{p} 2^{N p} \int_{G} \sup _{1 \leq n \leq 2^{N}}\left|\sum_{k=1}^{n} D_{k}(x)\right|^{p} d \mu(x) \leq c_{p} 2^{N(3 p-1)} .
\end{gathered}
$$

Lemma 3 is proved.

Lemma 4. Let $p \in(1 / 2,1]$. Then

$$
\int_{G}\left(\sup _{0 \leq q<2^{N}}\left|\sum_{k=q}^{2^{N}-1} D_{k}(x)(k-q+1)\right|\right)^{p} d \mu(x) \leq c_{p} 2^{N(3 p-1)} .
$$

Proof of Lemma 4. From Lemma 2 we have

$$
\begin{gathered}
\int_{G}\left(\sup _{0 \leq q<2^{N}}\left|\sum_{k=q}^{2^{N}-1} D_{k}(x)(k-q+1)\right|\right)^{p} d \mu(x) \\
\leq c_{p} 2^{N p} \int_{G} \sup _{0 \leq q<2^{N}}\left(\left|\sum_{k=0}^{q-1} D_{k}(x)\right|\right)^{p} d \mu(x) \\
+c_{p} \int_{G} \sup _{0 \leq q<2^{N}}\left(\left|\sum_{k=0}^{q-1}(k+1) D_{k}(x)\right|\right)^{p} d \mu(x) \leq c_{p} 2^{N(3 p-1)} .
\end{gathered}
$$

Lemma 4 is proved.

Let $\alpha:=\left(\alpha_{1}, \alpha_{2}\right): \mathbb{N}^{2} \rightarrow \mathbb{N}^{2}$ be a function. Define the following Marcinkiewiczlike kernels

$$
\frac{1}{n} \sum_{k=0}^{n-1} D_{\alpha_{1}(n, k)}(x) D_{\alpha_{2}(n, k)}(y), n \in \mathbb{P} .
$$

Denote by $\# B$ the cardinality of set $B$. Suppose that

(4) $\#\left\{l \in \mathbb{N}: \alpha_{j}(n, l)=\alpha_{j}(n, k), l<n\right\} \leq C \quad(k<n, n \in \mathbb{P}, j=1,2)$.

Lemma 5. Let $p \in(4 / 5,1]$. Then

$$
\sup _{1 \leq n \leq 2^{N}} \int_{\bar{I}_{N} \times \bar{I}_{N}}\left|\sum_{k=0}^{n-1} D_{\alpha_{1}(n, k)}(x) D_{\alpha_{2}(n, k)}(y)\right|^{p} d \mu(x, y) \leq c_{p} 2^{N(3 p-2)} .
$$


Proof of Lemma 5. In the sequel we use termonology and methods of paper [6]. Set

$$
n^{(s)}:=\sum_{k=s}^{\infty} n_{k} 2^{s}, J_{k}:=I_{k} \backslash I_{k+1} . \text { Thus } n^{(0)}=n, n^{(N)}=0, n, k \in \mathbb{N} .
$$

Then by

$$
\sum_{k=0}^{n-1} D_{\alpha_{1}(n, k)}(x) D_{\alpha_{2}(n, k)}(y)=\sum_{s=0}^{N-1} n_{s} \sum_{k=0}^{2^{s}-1} D_{\alpha_{1}\left(n, k+n^{(s+1)}\right)}(x) D_{\alpha_{1}\left(n, k+n^{(s+1)}\right)}(y)
$$

and $\bar{I}_{N}=\cup_{i=0}^{N-1} J_{i}=\cup_{i=0}^{N-1}\left(I_{i} \backslash I_{i+1}\right)$ we have

$$
\begin{aligned}
\int_{\bar{I}_{N} \times \bar{I}_{N}}\left|\sum_{k=0}^{n-1} D_{\alpha_{1}(n, k)}(x) D_{\alpha_{2}(n, k)}(y)\right|^{p} d \mu(x, y) \\
\leq \sum_{s=0}^{N-1} \int_{\bar{I}_{N} \times \bar{I}_{N}} \mid \sum_{k=0}^{2^{s}-1} D_{\alpha_{1}\left(n, k+n^{(s+1)}\right)} \\
\quad \times\left. D_{\alpha_{2}\left(n, k+n^{(s+1)}\right)}(y)\right|^{p} d \mu(x, y) \\
=\sum_{i=0}^{N-1} \sum_{j=i}^{N-1} \sum_{s=0}^{N-1} \int_{J_{i} \times J_{j}} \mid \sum_{k=0}^{2^{s}-1} D_{\alpha_{1}\left(n, k+n^{(s+1)}\right)}(x) \\
\quad \times\left. D_{\alpha_{2}\left(n, k+n^{(s+1)}\right)}(y)\right|^{p} d \mu(x, y) \\
+\sum_{i=0}^{N-1} \sum_{j=0}^{i-1} \sum_{s=0}^{N-1} \int_{J_{i} \times J_{j}} \mid \sum_{k=0}^{2^{s}-1} D_{\alpha_{1}\left(n, k+n^{(s+1)}\right)}(x) \\
\quad \times\left. D_{\alpha_{2}\left(n, k+n^{(s+1)}\right)}(y)\right|^{p} d \mu(x, y)=: A+B
\end{aligned}
$$

where $n \leq 2^{N}$. We can write

$$
\begin{aligned}
A= & \sum_{i=0}^{N-1} \sum_{j=i}^{N-1} \sum_{s=0}^{i-1} \int_{J_{i} \times J_{j}} \mid \sum_{k=0}^{2^{s}-1} D_{\alpha_{1}\left(n, k+n^{(s+1)}\right)}(x) \\
& \times\left. D_{\alpha_{2}\left(n, k+n^{(s+1)}\right)}(y)\right|^{p} d \mu(x, y) \\
& +\sum_{i=0}^{N-1} \sum_{j=i}^{N-1} \sum_{s=i}^{N-1} \int_{J_{i} \times J_{j}} \mid \sum_{k=0}^{2^{s}-1} D_{\alpha_{1}\left(n, k+n^{(s+1)}\right)}(x) \\
& \times\left. D_{\alpha_{2}\left(n, k+n^{(s+1)}\right)}(y)\right|^{p} d \mu(x, y) \\
= & : A_{1}+A_{2} .
\end{aligned}
$$


Since for every $n \in \mathbb{N}$ and $x \in J_{i}=I_{i} \backslash I_{i+1}$ we have $\left|D_{n}(x)\right| \leq c 2^{i}$ then we also have

$$
\left|D_{\alpha_{1}(n, k)}(x) D_{\alpha_{2}(n, k)}(y)\right| \leq c 2^{i+j},(x, y) \in J_{i} \times J_{j} .
$$

For $A_{1}$ this implies in the case of $1>(p>2 / 3)$

$$
A_{1} \leq c_{p} \sum_{i=0}^{N} \sum_{j=i}^{N} \sum_{s=0}^{i-1} \frac{2^{p(s+i+j)}}{2^{i+j}} \leq c_{p} 2^{N(3 p-2)} .
$$

If $p=1$, then for $A_{1}$ we have

$$
\begin{aligned}
A_{1} & \leq c_{1} \sum_{i=0}^{N} \sum_{j=i}^{N} \sum_{s=0}^{i-1} \frac{2^{s+i+j}}{2^{i+j}} \leq c_{1} \sum_{i=0}^{N} \sum_{j=i}^{N} 2^{i} \\
& \leq c_{1} \sum_{i=0}^{N}(N-i+1) 2^{i} \leq c_{1} 2^{N} \leq c_{p} 2^{N(3 p-2)} .
\end{aligned}
$$

That is, for every $(p>2 / 3)$

$$
A_{1} \leq c_{p} \sum_{i=0}^{N} \sum_{j=i}^{N} \sum_{s=0}^{i-1} \frac{2^{p(s+i+j)}}{2^{i+j}} \leq c_{p} 2^{N(3 p-2)} .
$$

Turn our attention to $A_{2}$. By (1) for $x \in J_{i}=I_{i} \backslash I_{i+1}$ we can write

$$
\begin{gathered}
D_{\alpha_{1}\left(n, k+n^{(s+1)}\right)}(x)=-w_{\left(\alpha_{1}\left(n, k+n^{(s+1)}\right)\right)^{(i+1)}}(x) \\
\times\left(\sum_{r=0}^{i-1}\left(\alpha_{1}\left(n, k+n^{(s+1)}\right)\right)_{r} 2^{r}-\left(\alpha_{1}\left(n, k+n^{(s+1)}\right)\right)_{i} 2^{i}\right) .
\end{gathered}
$$

Set

$$
\theta(s, k, i):=\sum_{r=0}^{i-1}\left(\alpha_{1}\left(n, k+n^{(s+1)}\right)\right)_{r} 2^{r}-\left(\alpha_{1}\left(n, k+n^{(s+1)}\right)\right)_{i} 2^{i} .
$$

Apply the Cauchy-Schwarz inequality and follow the method of [6]:

$$
\begin{gathered}
\int_{J_{i} \times J_{j}}\left|\sum_{k=0}^{2^{s}-1} D_{\alpha_{1}\left(n, k+n^{(s+1)}\right)}(x) D_{\alpha_{2}\left(n, k+n^{(s+1)}\right)}(y)\right|^{p} d \mu(x, y) \\
\leq \int_{J_{j}} 2^{-i(1-p / 2)}\left(\int_{J_{i}} \mid \sum_{k=0}^{2^{s}-1} D_{\alpha_{1}\left(n, k+n^{(s+1)}\right)}(x)\right. \\
\left.\times\left. D_{\alpha_{2}\left(n, k+n^{(s+1)}\right)}(y)\right|^{2} d \mu(x)\right)^{p / 2} d \mu(y)
\end{gathered}
$$




$$
\begin{gathered}
=\int_{J_{j}} 2^{-i(1-p / 2)}\left(\int_{J_{i}} \sum_{k, l=0}^{2^{s}-1} D_{\alpha_{1}\left(n, k+n^{(s+1)}\right)}(x)\right. \\
\left.\times D_{\alpha_{1}\left(n, l+n^{(s+1)}\right)}(x) D_{\alpha_{2}\left(n, k+n^{(s+1)}\right)}(y) D_{\alpha_{2}\left(n, l+n^{(s+1)}\right)}(y) d \mu(x)\right)^{p / 2} d \mu(y) \\
=\int_{J_{j}} 2^{-i(1-p / 2)}\left(\int_{J_{i}} \sum_{k, l=0}^{2^{s}-1} w_{\left(\alpha_{1}\left(n, k+n^{(s+1)}\right)\right)^{(i+1)}}(x)\right. \\
\times w_{\left(\alpha_{1}\left(n, l+n^{(s+1)}\right)\right)^{(i+1)}}(x) \theta(s, k, i) \theta(s, l, i) \\
\left.\times D_{\alpha_{2}\left(n, k+n^{(s+1)}\right)}(y) D_{\alpha_{2}\left(n, l+n^{(s+1)}\right)}(y) d \mu(x)\right)^{p / 2} d \mu(y) \\
=\int_{J_{j}} 2^{-i(1-p / 2)}\left(\sum_{k, l=0}^{2^{s}-1} \theta(s, k, i) \theta(s, l, i)\right. \\
\times D_{\alpha_{2}\left(n, k+n^{(s+1)}\right)}(y) D_{\alpha_{2}\left(n, l+n^{(s+1)}\right)}(y) \int_{J_{i}} w_{\left(\alpha_{1}\left(n, k+n^{(s+1)}\right)\right)^{(i+1)}(x)}(x / 2 \\
\left.\left.\times \alpha_{1}\left(n, l+n^{(s+1)}\right)\right)^{(i+1)}(x) d \mu(x)\right)^{p(y) .}
\end{gathered}
$$

Discuss the integral on the set $J_{i}=I_{i} \backslash I_{i+1}$

$$
\int_{J_{i}} w_{\left(\alpha_{1}\left(n, k+n^{(s+1)}\right)\right)^{(i+1)}}(x) w_{\left(\alpha_{1}\left(n, l+n^{(s+1)}\right)\right)^{(i+1)}}(x) d \mu(x) .
$$

If it differs from zero, then the $i+1$-th, $i+2$-th, $\ldots$ coordinates of $\alpha_{1}\left(n, n^{(s+1)}+\right.$ $k)$ and $\alpha_{1}\left(n, n^{(s+1)}+l\right)$ should be equal.

We have that for every $k$ there exists only a bounded numbers of $l$ 's for which $\alpha_{1}\left(n, n^{(s+1)}+k\right)=\alpha_{1}\left(n, n^{(s+1)}+l\right)$. These facts give that for every $k$ there exists - at most - $C 2^{i}$ number of l's for which this integral is not zero. This will be very important in the estimation of $A_{2}$ because - at first sight $k$ and $l$ are elements of $\left\{0,1, \ldots, 2^{s}-1\right\}$ and consequently this would mean $2^{2 s}$ addends. But this is not the case, because for every $k \in\left\{0,1, \ldots, 2^{s}-1\right\}$ the number of $l$ 's to be taken is $2^{i}$ because we need to take the integrals only when they are not zero. Thus, the number of $(k, l)$ pairs we have to take account is only $2^{s+i}$ and not $2^{2 s}$.

Consequently, apply the facts that $|\theta(s, k, i)| \leq c 2^{i},\left|D_{\alpha_{2}\left(n, k+n^{(s+1)}\right)}(y)\right| \leq$ $c 2^{j}$ we obtain 


$$
\begin{aligned}
& \int_{J_{j}} 2^{-i(1-p / 2)}\left(\sum_{k, l=0}^{2^{s}-1} \theta(s, k, i) \theta(s, l, i)\right. \\
& \times D_{\alpha_{2}\left(n, k+n^{(s+1)}\right)}(y) D_{\alpha_{2}\left(n, l+n^{(s+1)}\right)}(y) \int_{J_{i}} w_{\left(\alpha_{1}\left(n, k+n^{(s+1)}\right)\right)^{(i+1)}(x)} \\
& \left.\times w_{\left(\alpha_{1}\left(n, l+n^{(s+1)}\right)\right)^{(i+1)}}(x) d \mu(x)\right)^{p / 2} d \mu(y) \\
& \leq c \int_{J_{j}} 2^{-i(1-p / 2)}\left(2^{s+i} 2^{i} 2^{i} 2^{j} 2^{j}\right)^{p / 2} d \mu(y)=c \int_{J_{j}} 2^{-i(1-p / 2)}\left(2^{s+i} 2^{2 i+2 j+s}\right)^{p / 2} d \mu(y) .
\end{aligned}
$$

This gives that for $A_{2}$ we obtain

$$
\begin{aligned}
A_{2} & \leq c_{p} \sum_{i=0}^{N} \sum_{j=i}^{N} \sum_{s=i}^{N} \int_{J_{j}} 2^{-i(1-p / 2)}\left(2^{2 i+2 j+s}\right)^{p / 2} d \mu(y) \\
& \leq c_{p} 2^{N p / 2} \sum_{i=0}^{N} 2^{i(3 p / 2-1)} \sum_{j=i}^{N} 2^{j(p-1)} \\
& \leq c_{p} 2^{N p / 2} \sum_{i=0}^{N} 2^{i(5 p / 2-2)} \\
& \leq c_{p} 2^{N(3 p-2)}
\end{aligned}
$$

for $p \in(4 / 5,1)$. Let $p=1$. Then

$$
\begin{aligned}
A_{2} & \leq 2^{N / 2} \sum_{i=0}^{N} 2^{i / 2}(N-i+1) \\
& \leq 2^{N} \sum_{i=0}^{N} \frac{(N-i+1)}{2^{(N-i) / 2}} \leq c 2^{N} .
\end{aligned}
$$

Combining (15)-(10) we complete the proof of Lemma 5 .

Corollary 1. Let $\alpha_{1}(n, k)=k, \alpha_{2}(n, k)=n-k, k=0, \ldots, n-1, n \in \mathbb{P}$, $p \in(4 / 5,1]$. Then

$$
\sup _{1 \leq n \leq 2^{N}} \int_{\bar{I}_{N} \times \bar{I}_{N}}\left|\sum_{k=0}^{n-1} D_{k}(x) D_{n-k}(y)\right|^{p} d \mu(x, y) \leq c_{p} 2^{N(3 p-2)} .
$$


Corollary 2. Let $p \in(4 / 5,1]$. Then

$$
\sup _{0 \leq q<2^{N}} \int_{\bar{I}_{N} \times \bar{I}_{N}}\left|\sum_{k=q}^{2^{N}-1} D_{k}(x) D_{k-q}(y)\right|^{p} d \mu(x, y) \leq c_{p} 2^{N(3 p-2)} .
$$

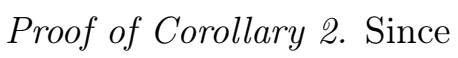

$$
w_{2^{N-1}} D_{j}=D_{2^{N}}-D_{2^{N}-j}, j=0,1, \ldots, 2^{N}-1
$$

from Lemma 2 and Corollary 1 we can write

$$
\begin{gathered}
\int_{\bar{I}_{N} \times \bar{I}_{N}}\left|\sum_{k=q}^{2^{N}-1} D_{k}(x) D_{k-q}(y)\right|^{p} d \mu(x, y) \\
\leq \int_{\bar{I}_{N} \times \bar{I}_{N}}\left|\sum_{k=0}^{2^{N}-q}\left(D_{2^{N}}(x)-D_{2^{N}-(k+q)}(x)\right) D_{k}(y)\right|^{p} d \mu(x, y) \\
\leq c_{p} \int_{\bar{I}_{N} \times \bar{I}_{N}} D_{2^{N}}(x)\left|\sum_{k=0}^{2^{N}-q} D_{k}(y)\right|^{p} d \mu(x, y) \\
+c_{p}\left|\sum_{\bar{I}_{N} \times \bar{I}_{N}}^{2^{N}-q} D_{k=0}^{p} D_{2^{N}-q-k}(x) D_{k}(y)\right|^{p} d \mu(x, y) \leq c_{p} 2^{N(3 p-2)} .
\end{gathered}
$$

Corollary 2 is proved.

It is well known that $D_{0}=0$ but to have symmetry, that is for some technical reason, at same places we have to indicate the 0th Dirichlet kernel in sums below.

Lemma 6. Let $n=2^{N} q_{1}+q_{2}, 0 \leq q_{2}<2^{N}$. Then

$$
\begin{aligned}
& K_{n}^{\Delta}(x, y)=\sum_{l=0}^{q_{1}-1} w_{l}\left(2^{N} x\right) w_{q_{1}-l}\left(2^{N} y\right) \sum_{k=1}^{q_{2}-1} D_{k}(x) D_{q_{2}-k}(y) \\
&+D_{2^{N}}(y) \sum_{l=0}^{q_{1}-1} w_{l}\left(2^{N} x\right) D_{q_{1}-l}\left(2^{N} y\right) \sum_{k=1}^{2^{N}} D_{k}(x) \\
&+D_{2^{N}}(x) \sum_{l=0}^{q_{1}-1} D_{l}\left(2^{N} x\right) w_{q_{1}-l}\left(2^{N} y\right) q_{2} K_{q_{2}}(y) \\
&+D_{2^{N}}(x) D_{2^{N}}(y) \sum_{l=0}^{q_{1}-1} D_{l}\left(2^{N} x\right) D_{q_{1}-l}\left(2^{N} y\right) 2^{N} \\
&-w_{2^{N}-1}(y) \sum_{l=0}^{q_{1}-1} w_{l}\left(2^{N} x\right) w_{q_{1}-l-1}\left(2^{N} y\right) \sum_{k=q_{2}+1}^{2^{N}} D_{k}(x) D_{k-q_{2}-1}(y)
\end{aligned}
$$




$$
\begin{aligned}
& -w_{2^{N}-1}(y) D_{2^{N}}(x) \sum_{l=0}^{q_{1}-1} D_{l}\left(2^{N} x\right) w_{q_{1}-l-1}\left(2^{N} y\right) \sum_{k=q_{2}+1}^{2^{N}} D_{k-q_{2}}(y) \\
& +w_{q_{1}}\left(2^{N} x\right) \sum_{k=1}^{q_{2}-1} D_{k}(x) D_{q_{2}-k}(y)+D_{2^{N}}(x) D_{q_{1}}\left(2^{N} x\right) \sum_{v=1}^{q_{2}-1} D_{v}(y) .
\end{aligned}
$$

Proof of Lemma 6. We can write

$$
\begin{aligned}
K_{n}^{\triangle}(x, y) & =\sum_{k=1}^{2^{N} q_{1}+q_{2}-1} D_{k}(x) D_{n-k}(y) \\
& =\sum_{k=1}^{2^{N} q_{1}} D_{k}(x) D_{n-k}(y)+\sum_{k=1}^{q_{2}-1} D_{k+2^{N} q_{1}}(x) D_{q_{2}-k}(y) \\
& =I_{1}+I_{2} .
\end{aligned}
$$

Since (see [2, (6.1)])

$$
D_{k+l 2^{N}}(x)=w_{l}\left(2^{N} x\right) D_{k}(x)+D_{2^{N}}(x) D_{l}\left(2^{N} x\right)
$$

for $I_{2}$ we have

$$
\begin{aligned}
I_{2}= & w_{q_{1}}\left(2^{N} x\right) \sum_{k=1}^{q_{2}-1} D_{k}(x) D_{q_{2}-k}(y) \\
& +D_{2^{N}}(x) D_{q_{1}}\left(2^{N} x\right) \sum_{k=1}^{q_{2}-1} D_{k}(y)
\end{aligned}
$$

For $I_{1}$ we have

$$
\begin{aligned}
I_{1}= & \sum_{l=0}^{q_{1}-1} \sum_{k=1}^{2^{N}} D_{k+l 2^{N}}(x) D_{2^{N} q_{1}+q_{2}-l 2^{N}-k}(y) \\
= & \sum_{l=0}^{q_{1}-1} \sum_{k=1}^{q_{2}} D_{k+l 2^{N}}(x) D_{2^{N}\left(q_{1}-l\right)+q_{2}-k}(y) \\
& +\sum_{l=0}^{q_{1}-1} \sum_{k=q_{2}+1}^{2^{N}} D_{k+l 2^{N}}(x) D_{2^{N}\left(q_{1}-l\right)+q_{2}-k}(y) \\
= & I_{11}+I_{12} .
\end{aligned}
$$


From (12) we get

$$
\begin{aligned}
I_{11}= & \sum_{l=0}^{q_{1}-1} w_{l}\left(2^{N} x\right) w_{q_{1-l}}\left(2^{N} y\right) \sum_{k=1}^{q_{2}} D_{k}(x) D_{q_{2}-k}(y) \\
& +D_{2^{N}}(y) \sum_{l=0}^{q_{1}-1} w_{l}\left(2^{N} x\right) D_{q_{1-l}}\left(2^{N} y\right) \sum_{k=1}^{q_{2}} D_{k}(x) \\
& +D_{2^{N}}(x) \sum_{l=0}^{q_{1}-1} D_{l}\left(2^{N} x\right) w_{q_{1-l}}\left(2^{N} y\right) q_{2} K_{q_{2}}(y) \\
& +D_{2^{N}}(x) D_{2^{N}}(y) \sum_{l=0}^{q_{1}-1} D_{l}\left(2^{N} x\right) D_{q_{1-l}}\left(2^{N} y\right) q_{2} .
\end{aligned}
$$

Turn our attention to $I_{12}$. From the simple calculation we can write $\left(q_{2}<k \leq 2^{N}, 0 \leq l<q_{1}\right)$

$$
\begin{aligned}
& D_{2^{N}\left(q_{1}-l\right)-\left(k-q_{2}\right)}(y) \\
= & D_{2^{N}\left(q_{1}-l-1\right)+2^{N}-\left(k-q_{2}\right)}(y) \\
= & D_{2^{N}\left(q_{1}-l-1\right)}(y)+w_{2^{N}\left(q_{1}-l-1\right)}(y) D_{2^{N}-\left(k-q_{2}\right)}(y) .
\end{aligned}
$$

Since

$$
D_{2^{N}-\left(k-q_{2}\right)}(y)=D_{2^{N}}(y)-w_{2^{N}-1}(y) D_{k-q_{2}}(y),
$$

we obtain

$$
\begin{aligned}
& D_{2^{N}\left(q_{1}-l\right)+q_{2}-k}(y) \\
= & D_{2^{N}}(y) D_{q_{1}-l-1}\left(2^{N} y\right)+w_{q_{1}-l-1}\left(2^{N} y\right) D_{2^{N}}(y) \\
& -w_{q_{1}-l-1}\left(2^{N} y\right) w_{2^{N}-1}(y) D_{k-q_{2}}(y) .
\end{aligned}
$$

Hence from (12), we have

$$
\begin{gathered}
I_{12}=D_{2^{N}}(y) \sum_{l=0}^{q_{1}-1} w_{l}\left(2^{N} x\right) D_{q_{1}-l-1}\left(2^{N} y\right) \sum_{k=q_{2}+1}^{2^{N}} D_{k}(x) \\
+D_{2^{N}}(y) D_{2^{N}}(x) \sum_{l=0}^{q_{1}-1} D_{l}\left(2^{N} x\right) D_{q_{1}-l-1}\left(2^{N} y\right)\left(2^{N}-q_{2}\right) \\
+D_{2^{N}}(y) \sum_{l=0}^{q_{1}-1} w_{l}\left(2^{N} x\right) w_{q_{1}-l-1}\left(2^{N} y\right) \sum_{k=q_{2}+1}^{2^{N}} D_{k}(x) \\
+D_{2^{N}}(y) D_{2^{N}}(x) \sum_{l=0}^{q_{1}-1} D_{l}\left(2^{N} x\right) w_{q_{1}-l-1}\left(2^{N} y\right)\left(2^{N}-q_{2}\right) \\
-w_{2^{N}-1}(y) \sum_{l=0}^{q_{1}-1} w_{l}\left(2^{N} x\right) w_{q_{1}-l-1}\left(2^{N} y\right) \sum_{k=q_{2}+1}^{2^{N}} D_{k}(x) D_{k-q_{2}}(y)
\end{gathered}
$$




$$
\begin{gathered}
-w_{2^{N}-1}(y) D_{2^{N}}(x) \sum_{l=0}^{q_{1}-1} D_{l}\left(2^{N} x\right) w_{q_{1}-l-1}\left(2^{N} y\right) \sum_{k=q_{2}+1}^{2^{N}} D_{k-q_{2}}(y) \\
=D_{2^{N}}(y) \sum_{l=0}^{q_{1}-1} w_{l}\left(2^{N} x\right) D_{q_{1}-l}\left(2^{N} y\right) \sum_{k=q_{2}+1}^{2^{N}} D_{k}(x) \\
+D_{2^{N}}(y) D_{2^{N}}(x) \sum_{l=0}^{q_{1}-1} D_{l}\left(2^{N} x\right) D_{q_{1}-l}\left(2^{N} y\right)\left(2^{N}-q_{2}\right) \\
-w_{2^{N}-1}(y) \sum_{l=0}^{q_{1}-1} w_{l}\left(2^{N} x\right) w_{q_{1}-l-1}\left(2^{N} y\right) \sum_{k=q_{2}+1}^{2^{N}} D_{k}(x) D_{k-q_{2}}(y) \\
-w_{2^{N}-1}(y) D_{2^{N}}(x) \sum_{l=0}^{q_{1}-1} D_{l}\left(2^{N} x\right) w_{q_{1}-l-1}\left(2^{N} y\right) \sum_{k=q_{2}+1}^{2^{N}} D_{k-q_{2}}(y) .
\end{gathered}
$$
6.

Combining (11), (14), (15) and (16) we complete the proof of Lemma

Lemma 7. Let $(x, y) \in \bar{I}_{N} \times \bar{I}_{N}$ and $n=2^{N} q_{1}+q_{2}, 0 \leq q_{2}<2^{N}$. Then the following inequality holds

$$
\begin{aligned}
& \int_{I_{N} \times I_{N}}\left|K_{n}^{\triangle}(x+s, y+t)\right| d \mu(s, t) \\
\leq & \frac{c}{2^{3 N}}\left\{\left|\sum_{k=0}^{q_{2}-1} D_{k}(x) D_{q_{2}-k}(y)\right|+\left|\sum_{k=q_{2}+1}^{2^{N}} D_{k}(x) D_{k-q_{2}}(y)\right|\right\} .
\end{aligned}
$$

Proof of Lemma [7. Since $x+s, y+t \notin I_{N}$, by lemma 6 we have

$$
\begin{aligned}
& n K_{n}^{\triangle}(x, y) \\
= & \sum_{l=0}^{q_{1}-1} w_{l}\left(2^{N} x\right) w_{q_{1}-l}\left(2^{N} y\right) \sum_{k=0}^{q_{2}-1} D_{k}(x) D_{q_{2}-k}(y) \\
& -w_{2^{N}-1}(y) \sum_{l=0}^{q_{1}-1} w_{l}\left(2^{N} x\right) w_{q_{1}-l-1}\left(2^{N} y\right) \sum_{k=q_{2}+1}^{2^{N}} D_{k}(x) D_{k-q_{2}}(y) \\
& +w_{q_{1}}\left(2^{N} x\right) \sum_{k=0}^{q_{2}-1} D_{k}(x) D_{q_{2}-k}(y) .
\end{aligned}
$$

Consequently $\left(q_{1} \leq n 2^{-N}\right)$

$$
\int_{I_{N} \times I_{N}}\left|K_{n}^{\triangle}(x+s, y+t)\right| d \mu(s, t)
$$




$$
\begin{aligned}
& \quad \leq \frac{1}{n}\left|\sum_{k=0}^{q_{2}-1} D_{k}(x) D_{q_{2}-k}(y)\right| \\
& \times \int_{I_{N} \times I_{N}}\left|\sum_{l=0}^{q_{1}-1} w_{l}\left(2^{N}(x+s)\right) w_{q_{1}-l}\left(2^{N}(y+t)\right)\right| \\
& \quad+\frac{1}{n}\left|\sum_{k=q_{2}+1}^{2^{N}} D_{k}(x) D_{k-q_{2}}(y)\right| \\
& \quad \int_{I_{N} \times I_{N}}\left|\sum_{l=0}^{q_{1}-1} w_{l}\left(2^{N}(x+s)\right) w_{q_{1}-l-1}\left(2^{N}(y+t)\right)\right| \\
& \leq \frac{c}{2^{3 N}}\left\{\left|\sum_{k=0}^{q_{2}-1} D_{k}(x) D_{q_{2}-k}(y)\right|+\left|\sum_{k=q_{2}+1} D_{k}(x) D_{k-q_{2}}(y)\right|\right. \\
& \leq \frac{1}{n 2^{2 N}}\left\{\left|\sum_{k=0}^{q_{2}-1} D_{k}(x) D_{q_{2}-k}(y)\right|\right.
\end{aligned}
$$

Lemma 7 is proved.

Lemma 8. Let $(x, y) \in \bar{I}_{N} \times I_{N}$ and $n=2^{N} q_{1}+q_{2}, 0 \leq q_{2}<2^{N}$. Then the following inequality holds

$$
\begin{aligned}
& \int_{I_{N} \times I_{N}}\left|K_{n}^{\triangle}(x+s, y+\dot{+} t)\right| d \mu(s, t) \\
\leq & \frac{c}{2^{3 N}}\left\{\left|\sum_{k=0}^{q_{2}-1} D_{k}(x) D_{q_{2}-k}(y)\right|+\left|\sum_{k=q_{2}+1}^{2^{N}} D_{k}(x) D_{k-q_{2}}(y)\right|\right. \\
& \left.+D_{2^{N}}(y)\left|\sum_{k=1}^{2^{N}} D_{k}(x)\right|\right\}, n \geq 2^{N} .
\end{aligned}
$$


Proof of Lemma 8. From (11) and Lemma 6 we get

$$
\begin{aligned}
& n K_{n}^{\triangle}(x, y) \\
= & \sum_{l=0}^{q_{1}-1} w_{l}\left(2^{N} x\right) w_{q_{1}-l}\left(2^{N} y\right) \sum_{k=0}^{q_{2}-1} D_{k}(x) D_{q_{2}-k}(y) \\
& +\left(\sum_{k=1}^{2^{N}-1} D_{k}(x)\right) D_{2^{N}}(y) \sum_{l=0}^{q_{1}-1} w_{l}\left(2^{N} x\right) D_{q_{1}-l}\left(2^{N} y\right) \\
& -w_{2^{N}-1}(y) \sum_{l=0}^{q_{1}-1} w_{l}\left(2^{N} x\right) w_{q_{1}-l-1}\left(2^{N} y\right) \sum_{k=q_{2}+1}^{2^{N}} D_{k}(x) D_{k-q_{2}}(y) \\
& +w_{q_{1}}\left(2^{N} x\right) \sum_{k=0}^{q_{2}-1} D_{k}(x) D_{q_{2}-k}(y) .
\end{aligned}
$$

Consequently,

$$
\begin{aligned}
& \int_{I_{N} \times I_{N}}\left|K_{n}^{\triangle}(x+s, y+t)\right| d \mu(s, t) \\
& \leq \frac{1}{n}\left|\sum_{k=0}^{q_{2}-1} D_{k}(x) D_{q_{2}-k}(y)\right| \\
& \times \int_{I_{N} \times I_{N}}\left|\sum_{l=0}^{q_{1}-1} w_{l}\left(2^{N}(x+s)\right) w_{q_{1}-l}\left(2^{N}(y+t)\right)\right| d \mu(s, t) \\
& +\frac{D_{2^{N}}(y)}{n}\left|\sum_{k=1}^{2^{N}-1} D_{k}(x)\right| \\
& \times \int_{I_{N} \times I_{N}}\left|\sum_{l=0}^{q_{1}-1} w_{l}\left(2^{N}(x+s)\right) D_{q_{1}-l}\left(2^{N}(y+t)\right)\right| d \mu(s, t) \\
& +\frac{1}{n}\left|\sum_{k=q_{2}}^{2^{N}-1} D_{k}(x) D_{k-q_{2}}(y)\right| \\
& \times \int_{I_{N} \times I_{N}}\left|\sum_{l=0}^{q_{1}-1} w_{l}\left(2^{N}(x \dot{+} s) x\right) w_{q_{1}-l-1}\left(2^{N}(y \dot{+} t)\right)\right| d \mu(s, t) \\
& +\frac{1}{2^{3 N}}\left|\sum_{k=0}^{q_{2}-1} D_{k}(x) D_{q_{2}-k}(y)\right| \\
& \leq \frac{q_{1}}{n 2^{2 N}}\left|\sum_{k=0}^{q_{2}-1} D_{k}(x) D_{q_{2}-k}(y)\right|
\end{aligned}
$$




$$
\begin{gathered}
+\frac{D_{2^{N}}(y)}{n 2^{2 N}}\left|\sum_{k=1}^{2^{N}-1} D_{k}(x)\right| \int_{G \times G}\left|\sum_{l=0}^{q_{1}-1} w_{l}(u) D_{q_{1}-l}(v)\right| d \mu(u, v) \\
+\frac{q_{1}}{n 2^{2 N}}\left|\sum_{k=q_{2}+1}^{2^{N}} D_{k}(x) D_{k-q_{2}}(y)\right| \\
+\frac{1}{2^{3 N}}\left|\sum_{k=0}^{q_{2}-1} D_{k}(x) D_{q_{2}-k}(y)\right| .
\end{gathered}
$$

Since

$$
\begin{aligned}
& \int_{G \times G}\left|\sum_{l=0}^{q_{1}-1} w_{l}(u) D_{q_{1}-l}(v)\right| d \mu(u, v) \\
\leq & \left(\int_{G \times G}\left|\sum_{l=0}^{q_{1}-1} w_{l}(u) D_{q_{1}-l}(v)\right|^{2} d \mu(u, v)\right)^{1 / 2} \\
= & \left(\int_{G} \sum_{l=0}^{q_{1}-1} D_{q_{1}-l}^{2}(v) d \mu(v)\right)^{1 / 2} \\
= & \sum_{l=0}^{q_{1}-1}\left(q_{1}-l\right) \leq q_{1},
\end{aligned}
$$

by (17) we complete the proof of Lemma 8 ,

\section{Proofs of main Results}

Proof of Theorem 1. By Lemma 1, the proof of theorem will be complete if we show that the operator $\sigma_{n}^{\triangle}$ is $p$-quasi-local for each $4 / 5<p \leq 1$ and bounded from $L_{\infty}(G \times G)$ to $L_{\infty}(G \times G)$.

First, we prove the boundedness from $L_{\infty}(G \times G)$ to $L_{\infty}(G \times G)$. Ee can write

$$
\begin{aligned}
& \int_{G \times G} \frac{1}{n}\left|\sum_{k=1}^{n-1} D_{k}(x) D_{n-k}(y)\right| d \mu(x, y) \\
= & \int_{\bar{I}_{|n|} \times \bar{I}_{|n|}} \frac{1}{n}\left|\sum_{k=1}^{n-1} D_{k}(x) D_{n-k}(y)\right| d \mu(x, y) \\
+ & \int_{\bar{I}_{|n|} \times I_{|n|}} \frac{1}{n}\left|\sum_{k=1}^{n-1} D_{k}(x) D_{n-k}(y)\right| d \mu(x, y)
\end{aligned}
$$




$$
\begin{aligned}
& +\int_{I_{|n|} \times \bar{I}_{|n|}} \frac{1}{n}\left|\sum_{k=1}^{n-1} D_{k}(x) D_{n-k}(y)\right| d \mu(x, y) \\
& +\int_{I_{|n|} \times I_{|n|}} \frac{1}{n}\left|\sum_{k=1}^{n-1} D_{k}(x) D_{n-k}(y)\right| d \mu(x, y)
\end{aligned}
$$

From Corollary 1 we have

$$
\sup _{n} \int_{\bar{I}_{N} \times \bar{I}_{N}} \frac{1}{n}\left|\sum_{k=0}^{n-1} D_{k}(x) D_{n-k}(y)\right| d \mu(x, y)<\infty .
$$

Using Lemma 3 for $p=1$ we have

$$
\begin{gathered}
\int_{\bar{I}_{|n|} \times I_{|n|}} \frac{1}{n}\left|\sum_{k=1}^{n-1} D_{k}(x) D_{n-k}(y)\right| d \mu(x, y) \\
=\frac{1}{n 2^{|n|}} \int_{G}\left|\sum_{k=1}^{n-1}(n-k) D_{k}(x)\right| d \mu(x, y) \leq c<\infty, n \in \mathbb{P} .
\end{gathered}
$$

Analogously, we can prove that

$$
\sup _{n} \int_{I_{|n|} \times \bar{I}_{|n|}} \frac{1}{n}\left|\sum_{k=1}^{n-1} D_{k}(x) D_{n-k}(y)\right| d \mu(x, y)<\infty .
$$

From a simple calculation we have

$$
\begin{aligned}
& \int_{I_{|n|} \times I_{|n|}} \frac{1}{n}\left|\sum_{k=1}^{n-1} D_{k}(x) D_{n-k}(y)\right| d \mu(x, y) \\
\leq & \frac{1}{2^{3 N}} \sum_{k=1}^{n-1} k(n-k) \leq c<\infty .
\end{aligned}
$$

Combining (18)-(22) we conclude that

$$
\sup _{n} \int_{G \times G} \frac{1}{n}\left|\sum_{k=1}^{n-1} D_{k}(x) D_{n-k}(y)\right| d \mu(x, y)<\infty .
$$

Hence, from (2) and (3) we conclude that

$$
\left\|\sigma_{n}^{\triangle} f\right\|_{\infty} \leq c\|f\|_{\infty} .
$$

Now, we prove that $\sigma_{n}^{\triangle}$ is $p$-quasi-local. We considere three casses. Let $a$ be an arbitrary atom with support $I_{N}(u) \times I_{N}(v)$. It is easy to see that $\sigma_{n}^{\triangle}(a)=0$ if $n<2^{N}$. Therefore we can suppose that $n \geq 2^{N}$. Since the 
dyadic addition is a measure preserving group operation, we may assume that $u=v=0$.

Step 1. Integrating over $\bar{I}_{N} \times \bar{I}_{N}$. Since $\|a\|_{\infty} \leq c^{2 N / p}$ from Lemma 7 we obtain

$$
\begin{gathered}
\left|\sigma_{n}^{\triangle} a(x, y)\right| \leq\|a\|_{\infty} \int_{I_{N} \times I_{N}}\left|K_{n}^{\triangle}(x+s, y+t)\right| d \mu(s, t) \\
\leq \frac{c 2^{2 N / p}}{2^{3 N}}\left|\sum_{k=1}^{q_{2}-1} D_{k}(x) D_{q_{2}-k}(y)\right| \\
+\frac{c 2^{2 N / p}}{2^{3 N}}\left|\sum_{k=q_{2}+1}^{2^{N}-1} D_{k}(x) D_{k-q_{2}}(y)\right|, n=2^{N} q_{1}+q_{2}, 0 \leq q_{2}<2^{N} .
\end{gathered}
$$

Applying the inequality

$$
\left(\sum_{k} a_{k}\right)^{p} \leq \sum_{k} a_{k}^{p}, \quad(0<p \leq 1)
$$

by (23) we have

$$
\begin{aligned}
& \left|\sigma_{n}^{\triangle} a(x, y)\right|^{p} \\
\leq & \frac{c_{p} 2^{2 N}}{2^{3 N p}}\left\{\left(\left|\sum_{k=1}^{q_{2}-1} D_{k}(x) D_{q_{2}-k}(y)\right|^{p}+\left|\sum_{k=q_{2}+1}^{2^{N}-1} D_{k}(x) D_{k-q_{2}}(y)\right|^{p}\right)\right\} .
\end{aligned}
$$

Consequently, by Corollary 1 and Corollary 2 we can write

$$
\begin{aligned}
& \int_{\bar{I}_{N} \times \bar{I}_{N}}\left|\sigma_{n}^{\triangle} a(x, y)\right|^{p} d \mu(x, y) \\
\leq & \frac{c_{p} 2^{2 N}}{2^{3 N p}}\left\{\sup _{1 \leq q_{2} \leq 2^{N}} \int_{\bar{I}_{N} \times \bar{I}_{N}}\left|\sum_{k=1}^{q_{2}-1} D_{k}(x) D_{q_{2}-k}(y)\right|^{p} d \mu(x, y)\right. \\
& \left.+\left.\sup _{1 \leq q_{2} \leq 2^{N}} \int\left|\sum_{\bar{I}_{N} \times \bar{I}_{N}}\right| \sum_{k=q_{2}+1}^{2^{N}-1} D_{k}(x) D_{k-q_{2}}(y)\right|^{p} d \mu(x, y)\right\} \\
\leq & c_{p}<\infty, \quad 4 / 5<p \leq 1 .
\end{aligned}
$$


Step 2. Integrating over $\bar{I}_{N} \times I_{N}$. From Lemma 8 we obtain

$$
\begin{aligned}
& \left|\sigma_{n}^{\triangle} a(x, y)\right| \\
\leq & \|a\|_{\infty} \int_{I_{N} \times I_{N}}\left|K_{n}^{\triangle}(x+s, y \dot{+} t)\right| d \mu(s, t) \\
\leq & \frac{c 2^{2 N / p}}{2^{3 N}}\left\{\left|\sum_{k=1}^{q_{2}-1} D_{k}(x) D_{q_{2}-k}(y)\right|+\left|\sum_{k=q_{2}+1}^{2^{N}-1} D_{k}(x) D_{k-q_{2}}(y)\right|\right. \\
& \left.+D_{2^{N}}(y)\left|\sum_{k=1}^{2^{N}-1} D_{k}(x)\right|\right\} .
\end{aligned}
$$

Consequently, from Lemma 2, Lemma 3 and Lemma 4, we get

$$
\begin{aligned}
& \int_{\bar{I}_{N} \times I_{N}}\left|\sigma_{n}^{\triangle} a(x, y)\right|^{p} d \mu(x, y) \\
\leq & \frac{c_{p} 2^{N}}{2^{3 N p}}\left\{\int_{\bar{I}_{N}} \sup _{1 \leq q_{2} \leq 2^{N}}\left|\sum_{k=1}^{q_{2}-1} D_{k}(x)\left(q_{2}-k\right)\right|^{p} d \mu(x)\right. \\
& +\int_{\bar{I}_{N}} \sup _{1 \leq q_{2} \leq 2^{N}}\left|\sum_{k=q_{2}}^{2^{N}-1} D_{k}(x)\left(k-q_{2}\right)\right|^{p} d \mu(x) \\
& \left.+2^{N p} \int\left|\sum_{k=1}^{2^{N}-1} D_{k}(x)\right|^{p} d \mu(x)\right\} \\
\leq & \frac{c_{p} 2^{N}}{2^{3 N p}} 2^{(3 p-1) N}=c_{p}<\infty(4 / 5<p \leq 1) .
\end{aligned}
$$

Step 3. Integrating over $I_{N} \times \bar{I}_{N}$. The case is analogous to step 2 and we have

$$
\int_{I_{N} \times \bar{I}_{N}}\left|\sigma_{n}^{\triangle} a(x, y)\right|^{p} d \mu(x, y) \leq c_{p}<\infty \quad(4 / 5<p \leq 1) .
$$

Combining (24), (25) and (26) we complete the proof of Theorem 1 .

\section{REFERENCES}

[1] J. Fine, Cesàro summability of Walsh-Fourier series, Proc. Nat. Acad. Sci. U.S.A. 41 (1955), 588-591.

[2] J. Fine, On the Walsh functions, Trans. Amer. Math. Soc. 65:3 (1949), 372-414.

[3] N. J. Fujii, A maximal inequality for $H^{1}$-functions on a generalized Walsh-Paley group, Proc. Amer. Math. Soc. 77:1 (1979), 111-116. 
[4] G. Gát, U. Goginava and G. Tkebuchava, Convergence in measure of logarithmic means of quadratical partial sums of double Walsh-Fourier series. J. Math. Anal. Appl. 323:1 (2006), 535-549.

[5] G. Gát, U. Goginava and K. Nagy, On the Marcinkiewicz-Fejér means of double Fourier series with respect to the Walsh-Kaczmarz system. Studia Sci. Math. Hungar. 46:3 (2009), 399-421.

[6] G. Gát, On almost everywhere convergence and divergence of Marcinkiewicz-Like means of integrable functions with respect to the two-dimensional Walsh system, Journal of Approx. Theory 164:1 (2012), 145-161.

[7] U. Goginava, Marcinkiewicz-Fejer means of $d$-dimensional Walsh-Fourier series. J. Math. Anal. Appl. 307:1 (2005), 206-218.

[8] U. Goginava, The maximal operator of Marcinkiewicz-Fejer means of the $d$ dimensional Walsh-Fourier series, East J. Approx. 12:3 (2006), 295-302.

[9] U. Goginava, Maximal operators of $(C, \alpha)$-means of cubic partial sums of $d$ dimensional Walsh-Fourier series. Anal. Math. 33:4 (2007), 263-286.

[10] U. Goginava, The weak type inequality for the Walsh system, Studia Math. 185:1 (2008), 35-48.

[11] U. Goginava, The weak type inequality for the maximal operator of the Marcinkiewicz-Fejér means of the two-dimensional Walsh-Fourier series. J. Approx. Theory 154:2 (2008),161-180.

[12] U. Goginava and F. Weisz, Maximal operator of the Fejér means of triangular partial sums of two-dimensional Walsh-Fourier series. Georgian Math. J. 19:1 (2012),101115.

[13] H. Lebesgue, Recherches sur la convergence des series de fourier, Math. Ann. 61:2 (1905), 251-280.

[14] J. Marcinkiewicz, Sur une méthode remarquable de sommation des séries doubles de Fourier, Ann. Scuola Norm. Sup. Pisa Cl. Sci. (2) 8:2 (1939), 149-160.

[15] J. Marcinkiewicz and A. Zygmund. On the summability of double Fourier series, Fund. Math. 32 (1939), 122-132.

[16] F. Schipp, Über gewisse Maximaloperatoren, Ann. Univ. Sci. Budapest. Eotvos Sect. Math. 18 (1975), 189-195 (1976).

[17] F. Schipp, W. R. Wade, P. Simon and J. Pál, Walsh Series. An Introduction to Dyadic Harmonic Analysis, Adam Hilger, Ltd., Bristol, 1990.

[18] P. Simon, Investigations with respect to the Vilenkin system, Ann. Univ. Sci. Budapest. Eotvos Sect. Math. 27 (1984), 87-101 (1985).

[19] P. Simon, Cesàro summability with respect to two-parameter Walsh systems, Monatsh. Math. 131:4 (2000), 321-334.

[20] F. Weisz, Cesàro summability of one- and two-dimensional Walsh-Fourier series, Anal. Math. 22:3 (1996), 229-242.

[21] F. Weisz, Convergence of double Walsh-Fourier series and Hardy spaces, Approx. Theory Appl. (N.S.) 17:2 (2001), 32-44.

[22] F. Weisz, Summability of Multi-Dimensional Fourier Series and Hardy Spaces, Mathematics and its Applications, 541. Kluwer Academic Publishers, Dordrecht, 2002.

[23] F. Weisz, Triangular Cesàro summability of two-dimensional Fourier series, Acta Math. Hungar. 132 (2011), no. 1-2, 27-41

[24] F. Weisz, Cesàro-summability of higher-dimensional Fourier series. Ann. Univ. Sci. Budapest. Sect. Comput. 37 (2012), 47-64.

[25] L. Zhizhiashvili, Generalization of certain theorem of Marcinkiewicz, Izv. Akad. Nauk SSSR Ser. Mat. 32 (1968), 1112-1122 (in Russian); translation in Math. USSR, Izv. 2 (1968), 1065-1075.

[26] L. Zhizhiashvili, Trigonometric Fourier Series and Their Conjugates, Mathematics and its Applications, 372. Kluwer Academic Publishers Group, Dordrecht, 1996. 
[27] A. Zygmund, Trigonometric Series. Vol. I, II, Third edition. With a foreword by Robert A. Fefferman. Cambridge Mathematical Library. Cambridge University Press, Cambridge, 2002.

G. Gát, Institute of Mathematics and Computer Science, College of NyírEgyháza, P.O. Box 166, NyiregyháZA, H-4400 Hungary

E-mail address: gatgy@nyf.hu

U. Goginava, Department of Mathematics, Faculty of Exact and Natural Sciences, Tbilisi State University, Chavchavadze str. 1, Tbilisi 0128, Georgia

E-mail address: zazagoginava@gmail.com 